J. Egypt. Soc. Parasitol. (JESP),48(2),2018: $275-288$

\title{
TRAINING PROGRAM TO ENHANCE NURSE'S KNOWLEDGE, ATTITUDE AND PRACTICE REGARDING MALARIA IN A TANZANIAN MILITARY HOSPITAL By
}

\author{
MAHFOUZ AHMAD AL-AGROUDI ${ }^{1}$, LAILA ABD EL-MAWLA MEGAHED ${ }^{1}$, \\ LAWRENCE TIA BANDA ${ }^{2}$ AND TOSSON ALY MORSY ${ }^{3}$ \\ Military Medical Academy, Cairo11291 ${ }^{1}$, Nursing Directorate, Tanzania People's \\ Defence Forces ${ }^{2}$, and Department of Parasitology, Faculty of Medicine, \\ Ain Shams University, Cairo $11566^{3}$, Egypt
}

\section{Abstract}

Malaria is a life threatening disease caused by Plasmodium species. Five zoonotic species were identified of which the risky P. falciparum is encountered in tropical and sub-urban areas, but predominately in Africa. Between 2008 \& 2017, trend of malaria in Tanzania showed a decline in morbidity from 18 million cases per annum to 5.5 million.This study enhanced nursesknowledge, attitude and practice regarding malaria in a Tanzanian Military Hospital.

Design: A quasi- experimental study was used. Subjects were 106 nurses who accepted to participate in the study, the pilot study was done on 10 nurses and they were excluded from the study sample. Tools were (1) Characteristics of of subjects (2) Educational needs assessment. (3) Knowlegde questionnaire sheet (pre/post). (4) Observational checklist tool.

The results revealed that nuses knowledge, attitude, and practice were significantly improved, after implementation of the program that was obvious in comparing pre-test with post-test and in observational checklist scores.

Key words: Tanzania, Malaria, Military Nurses, Knowledge, Attitude, Practice

\section{Introduction}

The African continent continues to bear the greatest burden of malaria and greatest diversity of parasites, mosquito vectors, and human victims and evolutionary plasticity of malaria and their vectors was a major obstacle to eliminate diseasel (Ghansah et al, 2014). Military nursing staff especially those sharing with the International Peace-Keeping Missions were critical risky groups, and must have enough knowledge regarding arthropod-borne infectious diseases as well as communicable diseases (El Bahnawasy et al, 2014). In this aspect, malaria, which is endemic in Tanzania, is a mosquito-borne infectious disease affecting humans and other animals caused by parasiticprotozoan belonging to genus Plasmodium (Nadjm and Behrens, 2012). Malaria causes symptoms that typically include fever, tiredness, vomiting, and head-aches. In severe cases it can cause yellow skin, seizures, coma, or death (Snow et al, 2012). Besides, about 35million Tanzanianpopulations were at risk, pregnant women and under five children being the most vulnerable groups (de Savignyet al, 2004). Malaria continued to top the list of ten most threatening diseases to child survival in Tanzania (Mushiet al, 2016).It is also the leading cause of Tanzanian outpatients, inpatients, and admissions of children less than five years of age at health facilities (WHO, 2018).

This study aimed to enhance nursing staff knowledge, attitude and practice regarding malaria disease in a Military Tanzanian Hospital.

Study objectives: 1 . to assess Military Nursing Staff knowledge, attitude and practice regarding malaria. 2. to develop and implement training program regarding malaria. 3 . to evaluate effect of training program. 4. to design a self-instruction booklet on malaria. Research hypothesis: After program implementation military nursing staff knowledge would be improved.

\section{Subject and Methods}

The study enhanced nurses' knowledge, attitude and practice regarding malaria in a Tanzanian Military Hospital. Implementa- 
tion of the training program of nurse's knowledge, attitude and practice regarding malaria would significantly improve and in turn provide high quality nursing care to malaria patients and help in malaria prevention. Technical design: This involved research design, setting, subjects and tools.

Research design: A quasi experimental study design was used.

Study Setting: Study was conducted in Dar es Salaam Military Hospital in Tanzania. It is a general military referral hospital, provides inpatient and outpatient services to military personnel and their families as well as civilians. It includes departments of Medical, Surgical, Orthopaedic, Neuro-surgery, Ophthalmology, Ear Nose \& Throat (ENT) Paediatric, Obstetrics and Gynaecology, Inpatient, and Outpatient, Emergency, Radiology and Laboratory, with specialists and consultants for all branches.

Subjects: Nursing staff available $(n=106)$ in the study setting during data collection period and accepted to participate in the study.

Data collection tools were developed based on review of related literature and researches for the purpose of this study. It included: 1- Characteristics of nursing staff: Age, working unit, educational qualifications and years of experience. 2- Educational needs assessment questionnaire: Educational needs assessment questionnaire used to determine unmet educational needs of the nursing staff regarding malaria disease. 3Pre/post-test knowledge questionnaire: This part assessed the level of military nursing staff knowledge and attitude regarding malaria disease. It was used twice throughout the program. It included 27 multiple choice questions and 16true and false divided into the following 5 main dimensions: a- Definition, epidemics, causes, mode of transmission, incubation period of malaria, people at risk of acquiring malaria, and affecting organs. b- Signs and symptoms, diagnosis, laboratory tests, treatment and complications. c- Knowledge and practice (Nursing care). d- Malaria prevention. 4- Observational checklist: Based on educational program knowledge, the observational checklist was designed for the purpose of measuring the military nursing staff's attitude and practice levels.

Part-2: A questionnaire to determine educational needs of military nursing team on malaria. Mark correct answer (yes or no): 1Have you attended any training program on malaria? 2- Have you ever been involved/ conducted taking care of patient with malaria?

Part- 3: Knowledge Assessment (select 1 out of 4): 1. Malaria is a disease caused by:a parasite, virus, bacteria or all. 2. Malaria is endemic inAfrica: South and Central America, parts of Middle East or Asia. 3. Malaria is transmitted by: touching infected patient, contaminated water, female Anopheles bite or cough and sneezing. 4. Avoid malaria: wearing long parts and sleeves; minimize outdoors stay, taking vaccine or taking ant malarial medicine.5. Persons at risk and dying from malaria are heavily exposed to: bites of female Anopheles with falciparum, young children, pregnant women, or all. 6 . Symptoms include shaking chills, headache and fever, anaemia and jaundice, nausea, vomiting and diarrhoea or all. 7. A person feels sick after bitten by infected female Anopheles?10 days to 4 weeks, 7 days, One year or10 days to 4 weeks, 7 days. 8 . Visit health care provider for medication before travelling: 1 to 2 weeks, 2 to 3 days, 4 to 6 weeks, or just one day.9. Serious malaria pregnancy include: prematurity, miscarriage, still birth or all. 10. Diagnosis: clinical signs and symptoms, stained blood films, urine analysis or clinical signs and symptoms, stained blood films. 11. Preventive measures are except: Limit exposed skin and apply insect repellents for skin, wear dark colored clothes, permethrin impregnated clothes or sleep under net impregnated with permethrin.12. Incubation period of malaria?: less than 7 days, less than two months, between 7 days and 2 year or 3 days. 13. Most affected organ by mala- 
ria is heart, brain, small intestine, or liver. 14 . Where is most common malaria in Africa?: Southern, Central, Western, and/or Northern Africa. 15. One nosocomial infection by blood transfusion, infected water \& food, touching infected person, or touching infected supplies. 16. For malaria patient nurse must monitor vital signs, high blood pressure, difficulty speech, or all. 17. Complicated severe malaria laboratory tests are accept: severe anaemia, T3 \& T4 and liver function test, hypoglycaemia, or acidosis and renal impairment. 18. Nursing diagnosis for malaria are except: Impaired circulation, hyperthermia and fluid deficit, hyperthermia and over load fluid, or imbalanced nutrition less than body requirement. 19. Nurse must do for malaria patients except: assess patient airway, breathing and respiratory condition, monitor vital signs, assess skin colour, pulse and capillary, or give antipyretic immediately for feverish patient. 20. Patient with impaired circulation needs except supplement oxygen, well ventilated room, elevate bed's head 60 degree, or less in activities. 21. Hyperthermic patient nurse must monitoring frequently body temperature, administer prescribed antipyretic drugs for high temperature, use iced water compresses on for head and axilla, or all. 22. To maintain fluid balance for amalaria patient nurse must: give him fluids as he needs, administer parenteral fluids as ordered, monitor intake and output, or administer parenteral fluids as ordered, monitor intake and output. 23. Patient must return to doctor become feverish within: 2 months after treatment, 2 years, 3 years, or all. 24. Malaria patient must be advised to take full course of medication, to stop medication when fever sub side, to repeat medication if signs and symptoms continue, or none. 25. Patient suffers from vomiting nurse gives him an antiemetic medication from half an hour to 1 hour before antimalarial drugs, orders to stop taking medication, gives him another dose after vomiting, or gives him drug after vomiting from 1 to 2 hours. 26. Patient is suffering from fever, nurse gives him: Medication on time, antipyretics medication from 1 to 2 hours before taking antimalarial medicine, from 3 to 4 hours, or from 1 to 2 hours. 27. Patient must restfrom 1 to 2 hours after medication to avoid: dizziness, vomiting, hypotension, or all.

Mark correct answer (true or false):1. Malaria spreads from person to person like influenza. 2. Malaria is asexually transmitted? 3. Travellers must be vaccinated:2 weeks before departure to a malaria endemic country.4. Travellers confusing malaria symptoms with common cold or flu. 5. Fever is a symptom? 6. Dizziness is a symptom? 7. Blurred vision is a symptom? 8. Sweat is a symptom? 9. Fatigue is a symptom? 10. Splenomegaly is a symptom? 11. Does malignant malaria cause same complications as a toxoplasmosis pregnant woman? 12. Is malignant malaria pregnant woman is a legitimate cause of abortion? 13.Is syringe contaminated with patient blood transmits malarial infection?14. Doespatients' room can be sprayed with insecticides? 15. Does patient room can be sprayed with insect repellents? 16. Is wire gauze preventsmosquito passage to mosquito net or patient room?

Practice observation check list for nursing care for patient; done or not done (Tab. 7).

Scoring systems: For each question in the questionnaires and the observational checklist, the participant was granted one score for correct answer, and zero score for wrong one. Score was summed up and the total divided by the number of the items, given a mean score for the part these score were converted into a $\%$ score and computed.

Validity test: The developed questionnaire-res and program content were critically selected to ensure relevancy, clarity, readability, simplicity, and questionnaires sequence and completion time.

Operational design: Design involved description of the preparatory phase of the pilot and the actual field work.

Preparatory phase: Study reviewed related national and international literature concerning the topic of the study using text books, 
articles, journals and bulletins, in order to be more acquainted with the study area which enabled preparation of the literature review and finalization of data collection tools and developing training program. Data collection tools were reviewed for the final validation, modifications and recommendations.

Pilot study: Once official permission was granted the pilot study conducted on 10 military nurses working in Dar es Salaam Military Hospital and they were excluded from the study sample. Tools were assessed for clarity, applicability and feasibility. Also, estimated time needed for data collection, tools were finalized based on the pilot's findings.

Planning phase: Upon securing official permission the researcher started data collection process from the military nursing staff using the respective tools to make an initial assessment training program to enhance the Military Nurse's knowledge, attitude and practice regarding malaria in Tanzania. Based on the findings of this assessment and literature, the study developed training program framework set general and specific objectives allocate program resources and facilities, determine program strategy (sessions' time table) teaching methods, media to be used, learner activities and evaluation methods, teaching places and educational handout.

Training program was intended to enhance military nurse's knowledge, attitude and practice regarding malaria. By the end of the educational program nurses wouldbe able to understand the re-emerging malaria: 1. Definition, epidemics, causes, mode of transmission, and people at risk. 2. Incubation period signs and symptoms, affected organs of malaria. 3. Complications, diagnoses, and treatment of malaria.4. Nursing care of malaria patient. 5. Prevention of malaria.

Implementation phase: Training program included illustrative lectures, group discussions and video films and hand-outs. It was implemented in form of six training sessions;two sessions/week. Each session took one hour. Questionnaires sheet were distributed immediately before the first session only (pre-test), and at the last session end (post-test) for collecting data purpose. After three months the observational checklist was conductedto observe nurses during their work with patients at different stages from admission to discharge at different shifts to ascertain long term-effect and sustainability of the program.

Administrative design: An official permission was secured to conduct the study in a Tanzanian Military Hospital. The aim was explained to the director of hospital and nursing leaders to obtain consent and cooperation. Nurses were notified formality about training program.

Ethical considerations: At the beginning of the study an official approval was kindly allowed from the Egyptian Military Medical Academy. Before applying study the ethical clearance from the Scientific Research, Ethical of the director of the Military Institute of Health and Epidemiology was obtained. Also, permission was obtained from the Chief of Medical Services in Tanzania Peoples' Defence Forces to conduct the study at a Military Hospital through the General Officer Commanding the Military Hospital.

The participated nurses were assured that confidentiality, anonymity, and the right to withdraw from the study would be guaranteed to anyone at any time, in addition ethical approval letters were clarified in the study.

Statistical design: Data were computerized and analysed using SPSS-version 22. Qualitative data were described as frequency (no.) and percentage. Comparisons between preand post-interventions for each question was done using chi-square test for significance. $\mathrm{P}$-value equal or less than 0.005 was significant. 


\section{Results}

The results are shown in tables $(1,2,3,4,5,6 \& 7)$ and figures $(1,2,3,4,5,6,7 \& 8)$.

Table 1: Characteristics of studied Nursing Staff $(\mathrm{N}=96)$

\begin{tabular}{|l|c|c|}
\hline Demographic characters & No. & $\%$ \\
\hline Age (years):Less than 20 years & 4 & 4.17 \\
\hline More than 20 - Less than 30 & 26 & 27.08 \\
\hline More than 30 - Less than 40 & 27 & 28.13 \\
\hline More than 40 & 39 & 40.62 \\
\hline Male & 39 & 40.62 \\
\hline Female & 57 & 59.38 \\
\hline Working Unit: Emergency department & 6 & 6.25 \\
\hline Operating room & 12 & 12.5 \\
\hline Critical care unit & 9 & 9.37 \\
\hline Other units & 69 & 71.88 \\
\hline Educational Qualification: Certificate in Nursing & 35 & 36.46 \\
\hline Dinloma in Nursing & 44 & 45.83 \\
\hline Bachelor of Science in Nursing & 15 & 15.63 \\
\hline Master degree & 2 & 2.08 \\
\hline Years of experience in nursing: Less than 5 vears & 25 & 26.04 \\
\hline Less than 10 vears & 21 & 21.88 \\
\hline From 10 to 15 vears & 18 & 18.75 \\
\hline More than 15 vears & 32 & 33.33 \\
\hline
\end{tabular}

Table 2: Educational needs assessment of Nursing Staff regarding malaria.

\begin{tabular}{|l|c|c|}
\hline \multicolumn{1}{|c|}{ Items } & Yes & No \\
\hline Have you attended any training program on malaria? & $15(15.63 \%)$ & $81(84.37 \%)$ \\
\hline Have you ever been involved/conducted taking care of patient with malaria? & $82(85.42 \%)$ & $14(14.58 \%)$ \\
\hline
\end{tabular}

Table 3:Nursing staff knowledge regarding items in corrected pre and post- tests.

\begin{tabular}{|l|l|l|l|l|l|l|l|}
\hline \multirow{2}{*}{ No. } & \multirow{2}{*}{ Item } & Pre & \multicolumn{3}{l|}{ Post } & \multicolumn{2}{l|}{ Pre \& Post } \\
\cline { 3 - 6 } & & No & $\%$ & No & $\mathrm{s} \%$ & $\boldsymbol{X}^{2}$ & $\mathrm{p}$ \\
\hline 1 & Malaria is asexually transmitted disease? & 32 & 33.33 & 54 & 56.33 & 0.76 & 0.257 \\
\hline 2 & Malaria is endemic in & 80 & 83.33 & 87 & 90.62 & 14.42 & 0.007 \\
\hline 3 & Where is malaria most common within Africa & 27 & 28.12 & 74 & 77.08 & 16.8 & 0.060 \\
\hline 4 & Malaria is a disease caused by? & 96 & 100 & 96 & 100 & 0.00 & 0.001 \\
\hline 5 & One of the mode of transmission of malaria is & 66 & 68.75 & 92 & 95.83 & 8.9 & 0.046 \\
\hline 6 & Malaria spreads from person to person like influenza? & 64 & 66.67 & 92 & 95.83 & 7.3 & 0.001 \\
\hline 7 & Most persons at risk of getting very sick and dying from malaria are & 66 & 68.75 & 84 & 87.5 & 13.8 & 0.005 \\
\hline 8 & Most affected organ by malaria in your body is & 78 & 81.25 & 87 & 90.62 & 2.1 & 0.997 \\
\hline 9 & How soon will a person feel sick after being bitten by female infected mosquito? & 29 & 30.20 & 68 & 70.83 & 13.4 & 0.154 \\
\hline 10 & What is the incubation period of malaria? & 28 & 29.27 & 49 & 51.04 & 14 & 0.301 \\
\hline 11 & Syringe infected with blood of malaria patient can transmit malaria infection? & 39 & 40.63 & 46 & 47.92 & 0.247 & 0.399 \\
\hline 12 & Malaria transmitted by & 88 & 91.67 & 91 & 94.79 & 0.480 & 1.000 \\
\hline
\end{tabular}

Table 4:Nursing staff knowledge levels, regarding items in corrected pre and post- tests.

\begin{tabular}{|c|c|c|c|c|c|c|c|}
\hline \multirow{2}{*}{ No. } & \multirow{2}{*}{ Item } & \multicolumn{2}{|c|}{ Pre } & \multicolumn{2}{|c|}{ Post } & \multicolumn{2}{|c|}{ Pre \& Post } \\
\hline & & No & $\%$ & No & $\%$ & $X^{2}$ & $\mathrm{p}$ \\
\hline 1 & Symptoms of malaria include & 64 & 66.67 & 90 & 93.75 & 6.13 & 0.725 \\
\hline 2 & Fever a symptom of malaria? & 88 & 91.67 & 93 & 96.88 & 5.62 & 0.111 \\
\hline 3 & Dizziness a symptom of malaria? & 82 & 85.41 & 88 & 91.67 & 2.94 & 0.055 \\
\hline 4 & Blurred vision a symptom of malaria? & 58 & 60.41 & 89 & 92.71 & 0.03 & 0.854 \\
\hline 5 & Sweat is symptom of malaria & 52 & 54.16 & 85 & 88.54 & 1.83 & 0.182 \\
\hline 6 & Fatigue is a symptom of malaria? & 86 & 89.58 & 88 & 91.67 & 1.84 & 0.314 \\
\hline 7 & Enlarged spleen is a symptom of malaria? & 75 & 78.13 & 92 & 95.83 & 1.02 & 0.005 \\
\hline 8 & Malaria diagnosis is based on? & 61 & 63.54 & 94 & 97.92 & 5.94 & 0.039 \\
\hline 9 & Complicated severe malaria laboratory tests include all following except & 39 & 40.63 & 93 & 96.88 & 8.70 & 0.029 \\
\hline 10 & Serious pregnancy problems, that increased by malaria disease include & 77 & 80.21 & 84 & 87.5 & 17.70 & 0.000 \\
\hline 11 & Does malignant malaria cause complications as in toxoplasmosis pregnant women? & 53 & 55.21 & 62 & 64.58 & 4.20 & 0.041 \\
\hline 12 & Is a pregnant women with malignant malaria is a legitimate cause of abortion? & 40 & 41.67 & 90 & 93.75 & 1.31 & 0.254 \\
\hline 13 & Travellers may confuse malaria symptoms with common cold or flu & 70 & 72.92 & 77 & 80.21 & 4.93 & 0.030 \\
\hline
\end{tabular}


Table 5:Nursing Staff knowledge and practice levels regarding (Nursing care) in corrected pre and post -tests.

\begin{tabular}{|c|c|c|c|c|c|c|c|}
\hline \multirow{2}{*}{ No. } & \multirow{2}{*}{ Item } & \multicolumn{2}{|c|}{ Pre } & \multicolumn{2}{|c|}{ Post } & \multicolumn{2}{|c|}{ Pre \& Post } \\
\hline & & No & $\%$ & No & $\%$ & $X^{2}$ & $\mathrm{p}$ \\
\hline 1 & For patient with malaria nurse must monitor & 86 & 89.58 & 93 & 96.88 & 0.67 & 0.986 \\
\hline 2 & Nursing diagnosis for malaria include all following except & 43 & 44.79 & 92 & 95.83 & 3.1 & 0.974 \\
\hline 3 & Nurse must do all the following for malaria patients except & 23 & 23.96 & 93 & 96.88 & 7.3 & 0.270 \\
\hline 4 & Patient with impaired circulation needs following except & 32 & 33.33 & 92 & 95.83 & 5.7 & 0.653 \\
\hline 5 & Nurse must do the following for hyperthermic patient & 59 & 61.45 & 92 & 95.83 & 8.01 & 0.112 \\
\hline 6 & To maintain fluid balance for patient with malaria the nurse must & 57 & 59.38 & 57 & 59.38 & 13.38 & 0.037 \\
\hline 7 & Patient must be told to return to doctor if become feverish within & 70 & 72.92 & 73 & 76.04 & 25.60 & 0.002 \\
\hline 8 & Malaria patient must be advised & 72 & 75.00 & 85 & 88.54 & 46.87 & 0.001 \\
\hline 9 & If patient is suffering from vomiting nurse must advise him to & 17 & 17.70 & 14 & 14.58 & 42.16 & 0.001 \\
\hline 10 & If patient is suffering from fever nurse must advise him to & 47 & 48.96 & 51 & 53.13 & 36.40 & 0.001 \\
\hline 11 & Patient must rest from 1 to 2 hours after taking medication because of following risks & 65 & 67.70 & 56 & 58.33 & 17.47 & 0.420 \\
\hline
\end{tabular}

Table 6: Nursing Staff knowledge levels of study regarding prevention of malaria in corrected pre and post- tests.

\begin{tabular}{|c|c|c|c|c|c|c|c|}
\hline \multirow{2}{*}{ No. } & \multirow{2}{*}{ Item } & \multicolumn{2}{|l|}{ Pre } & \multicolumn{2}{|c|}{ Post } & \multicolumn{2}{|c|}{ Pre \& Post } \\
\hline & & No & $\%$ & No & $\%$ & $X^{2}$ & $\mathrm{p}$ \\
\hline 1 & Methods used to prevent malaria & 86 & 89.58 & 93 & 96.88 & 0.67 & 0.986 \\
\hline 2 & Visit your health care provider to take medication during & 25 & 26.04 & 75 & 78.13 & 10.1 & 0.634 \\
\hline 3 & Malaria is prevented by the following measures except & 14 & 14.58 & 90 & 93.75 & 8.7 & 0.413 \\
\hline 4 & Travellers must be vaccinated two weeks before departure to a malaria country & 77 & 80.21 & 89 & 92.71 & 11.6 & 0.002 \\
\hline 5 & Doesmalaria patients'room sprayed with insecticides? & 68 & 70.83 & 88 & 91.67 & 0.3 & 0.588 \\
\hline 6 & Does malaria patients' room sprayed with insect repellents? & 42 & 43.75 & 92 & 95.83 & 9.4 & 0.264 \\
\hline 7 & Is wire gauze prevent passage of mosquitoes on mosquito net or in patient room? & 55 & 57.29 & 92 & 95.83 & 0.1 & 0.763 \\
\hline
\end{tabular}

Table 7: Practiceobservational check list frequency distribution of nursing staff.

\begin{tabular}{|c|c|c|}
\hline Observed item & No. & $(\%)$ \\
\hline \multicolumn{3}{|l|}{ History the nurse ask about } \\
\hline Patient travel to a malaria area & 96 & 100 \\
\hline Has a history of malaria & 96 & 100 \\
\hline Take any Antimalarial drugs prior to admission & 96 & 100 \\
\hline Has recently any medication & 96 & 100 \\
\hline Has received any blood transfusion & 84 & 87.5 \\
\hline Has worked in clinical laboratory with infected blood & 93 & 96.88 \\
\hline \multicolumn{3}{|l|}{ Physical examination } \\
\hline Assess level of unconscious using Glasgow coma scale & 20 & 20.83 \\
\hline Monitor vital sign & 96 & 100 \\
\hline Look for signs of anaemia and bleeding & 96 & 100 \\
\hline Prepare for blood transfusion & 96 & 100 \\
\hline Monitor for hypoglycaemia especially for pregnant women & 96 & 100 \\
\hline \multicolumn{3}{|l|}{ For patient with impaired circulation } \\
\hline Assess patient's airway, breathing and respiratory conditions & 96 & 100 \\
\hline Supplement of oxygen in severe condition & 4 & 4.17 \\
\hline Maintain a well-ventilated room & 96 & 100 \\
\hline Elevate the head of the bed 30 degree & 1 & 1.04 \\
\hline Lessen activities that require moderate to high activities & 96 & 100 \\
\hline Monitor vital signs & 96 & 100 \\
\hline Assess skin colour, pulse and capillary refill & 96 & 100 \\
\hline Treat fever with tepid sponging & 96 & 100 \\
\hline Monitor vital signs and keep an eye on increase and Changes in body temperature & 96 & 100 \\
\hline Monitor fluid intake and output & 96 & 100 \\
\hline Assess for gastro intestine tract symptoms & 96 & 100 \\
\hline Review disease process and therapy focusing on Patient concerns & 96 & 100 \\
\hline Maintain patient hygiene by making sure bathing and linen Cleanliness & 96 & 100 \\
\hline Report to doctor on any poor progress of patient & 96 & 100 \\
\hline Provide psychological support to patient as frequent as possible & 96 & 100 \\
\hline
\end{tabular}

\section{Discussion}

Undoubtedly, the Military Medical Staff all over the world, particularly the Nursing group are the critical risky group and must be involved in having at least the general knowledge concerning the VHFs andother 
fatal infectious arthropod-borne diseases (El-Bahnasawy et al, 2015).

The continued public health burden of malaria in Sub-Sahara is due to a combination of factors, including: 1- Increasing resistance of malarial parasites to chemotherapy, 2Increasing resistance of the Anopheles mosquito vector to insecticides, 3- Ecologic and climate changes, and 4- Increasing travel to malaria-endemic areas by non-immune travellers (Al-Agroudi et al, 2017a).

Consequently, this study enhanced nurse's knowledge, attitude and practice regarding malaria in a Tanzanian Military Hospital. This was achieved by assessing their knowledge, attitude and practice, designing and implementing an intervention training program for nurses to fill any identified gaps and assessing its effect on quality nursing care provided for malaria patients.

The first step in planning any professionnal development activity was to assess the learning needs of the target group or individual to determine the structure of the program in terms of objectives, content and activities. Learning needs must be clearly defined during the planning process (Lynore and DeSilets, 2007).

Lahti et al. (2014) in Finland reviewed the impact of electronic-learning (e-learning) on nurses' and nursing students' knowledge, skills and satisfaction. But, they were unable to demonstrate a statistical difference between groups in e-learning and traditional learning relating to the nurses' or student nurses' know-ledge, skills and satisfaction. Sinclair et al. (2015) in Australia stated that the technological innovation has not only impacted social change in recent years but has been the prime driver of educational transformation. The newest consumers of post-secondary education, the so-called 'digital natives', have come to expect education to be delivered in a way that increased usability and convenience. Health care professionals (HCPs) in the clinical setting, particularly those in rural and remote communities, were no different. Today's health work- force must have a professional responsibility to maintain competency in the practice by achieving a minimum number of hours of continuing professional development. Thus, the HCPs seeking the professional development opportunities are reliant on sourcing these independently according to individual learningneeds. However, difficulties existed in some health professionals' access to ongoing professional development opportunities, particularly those with limited access face-to-face education due to geographical isolation or for those not enrolled in a formal program of study.

The present study was divided into four parts in order to deal with the studied problem from all aspects.

Part-1 included personal data variables such as age, sex, units of work, educational qualification and years of experience. The main characteristics of the study sample has been divide into four aging groups; less than 20 years old, more than 20-less than 30 years old, more than 30 -less than 40 years old, and the highest number $40.62 \%$ was from the forth age group more than 40 years old. Nearly two third of the nurses included were females.

In Tanzania, nursing as a profession was only for females for many years and in recent years males started joining this profession. Nikki and Campos (2010) reported that nursing was a feminine job with excellence and men are still outpacing women in salary even in the United States. They added that in order to meet the expectations of the public, nurses must fully understand the duties associated with their position as set forth by not only governing bodies, but also the institutional policies and procedures affecting their practice. One particular duty that deserves significant emphasis is the requirement of complete and accurate documentation related to patient care, which includes what's done to and for the patient and how particular decisions about care are made. This article provided direction and guidelines for nurses when documenting care for 
patients. Wojczewskiet al. (2015) stated that many female physicians and nurses of African countries have chance for international migration that increased considerably over the past four decades for possibilities of employment, or marriage or a better standard of living (Benatar and Brock, 2011).

The present study showed that the vast majority of nurses were from different working units $71.88 \%$ other than emergency department, operating room and critical care, which could be explained that the number of nurses in these units were small. The diploma nursing school graduates were the highest number $45.83 \%$. In Tanzania, diploma nursing schools graduates are greater in numbers and their salaries are lesser than other nursing educational systems graduates. More than one third of nurses are experienced more than 15 years.

In an attempt to fill the identified gaps of the health professionals, Tanzania implemented system of the mid-level health workers (MLHWs) in relation to specific health service needs. However, the policy documents showed a marked lack of progress in health outcomes relating to Millennium Development Goal 4 and 5 in Tanzania (The United Republic of Tanzania, 2015).

Part-11 dealt with the educational needs assessment of the nursing staff regarding malaria:

The present study showed that the great majority of nurses $(81.37 \%)$ did not attend any similar training program in the past. However, $82.42 \%$ of nurses have been conducted in taking care of malaria patients. This could be explained by lack of an active training or educational departments in the hospital. According to WHO (2014) a critical mass of competent health workers is needed with adequate skills to diagnose and treat malaria in both the public and the private health sector. They should be supported by community education for early recognition and health-seeking behaviour to ensure early treatment and referral.

In Tanzania, Mubyaziet al. (2005) assessed the knowledge, attitudes and practices of these groups in relation to malaria control with emphasis on intermittent preventive treatment in pregnancy (IPTp) services. They reported that the successful implementation of the IPTp strategy in Tanzania depends on the proper planning of, and support to, the training of health staff and sustained sensitization of pregnant women at health facility and community levels about the benefits of the IPTp for the women and their unborn babies.

Nsimba (2006) assessed knowledge about drugs in the Tanzanian Mothers/Guardians of sick children under the age five years immediately after consulting clinicians and after receiving drugs from the dispenser. He used as stipulated by the WHO'stechnique about how to investigate drug use in health facilities. He recommended the need for educational intervention for prescribers (health care providers) on rational prescribing of drugs, such as antimalarial, antibiotics, injections, proper dispensing, and adequate labelling drugs in packets, while the dispensing time for the drugs was too short. $\mathrm{He}$ claimed a need to advise the Ministry of Health to develop health education programs on a regular basis for all health care providers and mothers/guardians of children in general public/rural communities on how to use/administer anti-malarial and other drugs at home. Also, Nsimba (2007) in Dar-esSalaam evaluated short term effects of one to one educational intervention approach, conducted with 40 drug sellers in order to improve the private sector's practices, compliance and performance in using the national treatment guidelines for malaria and other common childhood illnesses. He concluded that changing the private sectors' knowledge, behaviour and practices/ performance was a slow and difficult process. The intervention approach applied was feasible at districtlevel. He added that other alternative strategies were needed to influence drug sellers'/ dispensers' compliance and performance.

Manzi et al. (2009) in Dar es Salaam repo- 
rted the rationale, development and implementation of a district-based strategy for implementation of intermittent preventive treatment in infants (IPTi) for malaria and anaemia control. The strategy was integrated into existing systems as far as possible and well accepted by health staff. Time-and-motion studies documented that IPTi implementation took a median of 12.4 minutes (range 1.6-28.9) per nurse/vaccination clinic. They concluded that collaborative approach between the researchers and health staff effectively translated research findings into a strategy fit for the public health implementation.

Part-111 illustrated the distribution of knowledge pre and post test scores among studied sample regarding items definition, epidemics, causes, mode of transmission, incubation period of malaria, people at risk of acquiring malaria, and affecting organs. The results showed that nurse's pre-test scores were low to moderate. The lowest scores were in items related to: epidemics: where is malaria most common in Africa $28.12 \%$, incubation period: what is the incubation period $29.27 \%$ and how soon will a person feel sick after being bitten by female infected mosquito $30.2 \%$ and in mode of transmission: like, malaria is asexually transmitted disease $33.33 \%$ and syringeinfected with blood of patient with malarial can transmit malaria infection $40.63 \%$.

In the present study, the post-test revealed a significant positive impact of training sessions illuminated on the knowledge score of nurses except for item syringe infected with blood of malaria patient can transmit infection. However, the items answers showed statistically significance difference as malaria spread from person to person like influen$\mathrm{za}$ and malaria is a disease caused by protozoan blood parasite (P-value $0.001 \& 0.001$ ) respectively. Therefore, the implementation of the training program in present study had successfully influenced nursing knowledge.

This agreed with Fey and Milliner (2008) who reported that the lack of the experience RNs combined with the significant growth in key service areas, required managers to examine the traditional hiring and orientation processes at the Washington Hospital Centre. They recommended development of a competency-based framework for new graduate nurses into specialty nursing areas, including cardiology and high-risk obstetrics. Kanamori et al. (2011) identified the status of the nurses' performance in managing malaria in Tanzanian patients, to provide the baseline data before the training intervention. They recommended a training programme for nurses and provision of working aids, such as a dosage table and a wall chart.

In pre-test, the study revealed that nurses scores were low to moderate, the lowest scores were in items related to, laboratory test: Complicated sever the laboratory test include $40.63 \%$, and complications: is a pregnant woman suffering from malignant malaria is a legitimate cause of abortion $41.67 \%$, does malignant malaria cause the same complications as a toxoplasmosis for pregnant woman $55.21 \%$, and in symptoms item; sweat is a symptom of malaria $54.16 \%$. The post-test nurse's scores showed positive significant impact of the training program regarding signs and symptoms, diagnosis, laboratory test, tre-atment and complications. However, items: serious pregnancy problems that increased by malaria disease included enlarged spleen is a symptom of malaria had statistically significance difference (P-value $0.000 \& 0.005$ respectively). The present result agreed with Tweed and Tweed (2008). They assessed intensive care nurses' knowledge of pressure ulcers and the impact of an educational program on knowledge levels. They concluded that among the ICU registered nurses, knowledge levels of pressure ulcer prevention and management were good at baseline. Knowledge levels improved with an educational program but soon returned to baseline.

In the present study, distribution of knowledge pre and post test scores among studied sample, regarding items in nursing care: In pre- test the study revealed that nurse's sco- 
res were low in the majority of items such as, if a patient is suffering from vomiting nurse must advise him to $17.7 \%$, the nurse must do all the following for malaria patients except $23.96 \%$, patient with impaired circulation needs the following except $33.33 \%$, nursing diagnosis of malaria include all the following except $44.79 \%$. While in post-test there was positive significant impact of the training program. But, items of malaria patient must be advised and if the patient is suffering from fever the nurse must advise to show statistically significance difference (P-value $0.001 \& 0.001$ respectively). This agreed with El-Bahnasawyet al. (2010) in Egypt. Also, the present result agreed with a study done by Kanamoriet al. (2011) conducted at 19 government health facilities in four districts of Tanzania. In order to assess the performance of nurses, 60 procedures involved in the management of malariapatients were observed and evaluated. Additionally, the exit interviews were conducted with 60 malariapatients or caretakers upon leaving the health facilities. They concluded that nurses did not satisfy the required standard in the management of malariapatients. The weak areas identified by the study are the quinine IV administration and health education provided to patients. They added that; conducting a training programme for nurses and the provision of working aids, such as a dosage table and a wall chart, are recommended. Furthermore, in order to alleviate the incorrect dosage in intravenous quinine administration associated with dosage calculation, the possibility of adopting treatment drugs that can be administered by other dosage routes could be explored.

Measuring Nursing Staff knowledge levels of study regarding prevention of malaria in pre-test the lowest nurses scores were in; malaria is prevented by the following measures except $14.58 \%$, visit your health care provider to take medication during $26.04 \%$ and does the room of malaria patient sprayed with insect repellents $43.75 \%$. However, item of travelers must be vaccinated two weeks before their departure to a country where malaria was present, gave highly statistically significance difference (P-value < 0.002).

In the same line Singh et al. (2014) stated that; misconceptions about malaria transmission and its cause still exist. Knowledge about preventive measures does not necessarily translate into improvement in practices. There was a need for targeted educational programs to increase the communities' efforts to develop desirable attitude and practices regarding malaria and their participation for malaria control. In the same line with the study Bauchet al.(2015) mentioned that health care providers and residents generally reported consistent use of malaria preventive measures. However, maintaining and continuing to reduce malaria transmission will require ongoing education for both health care providers and residents to reinforce the importance of using preventive measures

The nurses knowledge scores were low in pre- test throughout the study it could be explained that because, there is no active training nursing departments or staff development plans, and without encouragement for nurse's self-study. This went with Ssekabira et al. (2008) who reported that; training of health workers and improving diagnostic capabilities were identified as potential avenues for improving malaria case management. The study observational results suggested a need for more integrated training approaches and supportive supervision.

Generally, the results confirmed positive impact of training program in enhancing the nurse's knowledge, attitude and practice regarding malaria. The nurses were interested in understanding and in getting more information about such fatal disease that had high prevalence in their country causing burden on health and on economy. Amron (2013) agreed with the result and suggested that malaria control could be significantly improved in rural areas, if the caregivers were 
adequately empowered by the appropriate health education intervention though change in attitude and belief might require the longer and persistent effort.

Furthermore agreement was with HanafiBojdet al. (2011) who reported that in order to intensify elimination activities we need to have a better understanding of the knowledge, attitudes and practices (KAP) of malaria prevention. They added thatthe knowledge, attitudes and practices of respondents in this survey about malaria control were good in some cases, but in general needed to be improved with educational programs. Another agreement with the present study was that of Olalekan and Adebukola (2015) they stated that in conclusion, an increase in knowledge scores was observed when preand post-training scores on malaria prevention and control were compared. Training session on malaria improved knowledge of malaria prevention and control among role model community care givers studied. Thus, adequate start off and the on-the-job training could help in improving knowledge, and possibly, the performance of community role model care givers towards a successful malaria control.

McConnell et al. (2012) in Canada addressed relationship between specific training programs and the knowledge acquirement found an actual significant positive effect on knowledge acquirement after taking relevant training sessions as long as the educational or the training program was run properly. They added that high performing examinees showed stronger evidence of accurate self-monitoring than did low performing individuals and reliability of the measures suggested that they have the potential to differentiate between individuals.

Part-1V illustrated assessment of nursing staff attitude and practice by observational checklist frequency distribution tothem. The vast majority of nurses had $100 \%$ scores in observation checklist items except these items, elevating head of bed, oxygen supplement, assess level of conscious and got low scores $1 \%, 4 \%, \& 20.8 \%$ respectively, this could be explained that they do not have the permission to give oxygen and nurses are not trained on Glasgow coma scale and they don't have enough knowledge on the degree of elevation of bed, but the observational checklist reflected a significant positive impact of training sessions. Thus, continuous education program in present study had successfully influenced the nursing knowledge, attitude and practice. This agreed with Suchitra and Lakshmi Devi (2007) in India assessed the knowledge, attitudes and practices among the health care workers (HCWs) on nosocomial infections. They concluded that the education gave a positive impact on retention of know-ledge, attitudes and practices in all staff categories. They reported the need to develop continuous education for all staff categories to reduce the incidence of the nosocomial infections, compliance with the interventions are mandatory. Also, Tweed and Tweed (2008) in New Zealand stated that the completion of educational program improved the level of knowledge to prevent and manage pressure ulcers were good initially and improved with an educationalprogram, but soon returned to baseline. Djadou et al. (2011) in Togo evaluated the knowledge, attitudes and practices of health care providers (HCPs) regarding prevention of mother-to-child transmission of HIV (PMTCT). They reported that know-ledge, attitudes and practices of HCPs in To-go regarding PMTCT were more or less fairly good. But, it showed several weaknesses that should be addressed by further training. Apouey and Picone (2014) in Sub-Saharan Africa reported that the social interactions in malaria preventive behaviours in the form of social multipliers effects of women's education and household wealth.

Al-Agroudi et al. (2017b) in Egypt stated that there should be mandatory educational and training programs about the arthropodborne infectious diseases as malaria for all military nurses who would share in the Peacekeeping Missions to Sub-Sahara Afri- 
ca. They added that nurses must be encouraged for self-study done by bonus, promotions or incentives.

\section{Conclusion}

The study findings led to the conclusion that staff nurses knowledge, attitude and practice are generally low to moderate regarding thefive main following dimensions of malaria diseaseDefinition, epidemics, causes, mode of transmission, incubation period of malaria, people at risk of acquiring; signs and symptoms, diagnosis, laboratory test, treatment and complications as well as the nursing careand prevention of malaria.After the implementation of a training program for staff nurses, the comparison between pre and post-test showed statistical significance and generally, the result have shown a significant impact of training sessions illuminated in the follow up checklist score of nurses.

\section{References}

Al-Agroudi, MA,Megahed, LA, Banda, LT, Morsy, TA, 2017a:An overview on malaria in Sub-Saharan with special reference to Tanzania. J. Egypt. Soc. Parasitol. 47, 2:273-92.

Al-Agroudi, MA, Megahed, LA, Ibrahim, AMA,Abdallah, EM, Morsy, TA, 2017b:An educational program on re-emerging malaria for nursing staff in a Military Fever Hospital. J. Egypt. Soc. Parasitol. 47, 3: 665-72.

Amron, OE, 2013: Impact of health education prevention practices among nursing mothers in

rural communities in Nigeria. Niger Med. J. 54:115-22.

Apouey, B, Picone, G, 2014: Social interactions and malaria preventive behavior's in SubSaharan Africa. Hlth. Econ. 23, 9:994-1012.

Benatar, S, Brock, G, 2011: Global Health and Global Health Ethics: Cam-bridge University Press.

Bauch, JA, Gu, JJ, Msellem, M, Mårtensson, A, Ali, AS, et al, 2015: Perception of malaria risk in a setting of reduced malaria transmission: A qualitative study in Zanzibar. Bull. Jimma Inst. Hlth. Sci. ISSN1025-9732 http/www. ncbi. nlm.nih.gov

deSavigny, D, Mayombana, C, Mwageni, E, Masanja, H, Minhaj A, et al, 2004: Care- seeking patterns for fatal malaria in Tanzania. Malar J. 3:27. doi: 10.1186/1475-2875-3-27.

Djadou, KE, Koffi, KS, Saka, B, Tépé, EM, Vinyo, DK, et al, 2011: Knowledge, attitudes and practices of healthcare providers in Togo regard-ing prevention of mother-to-child transmission of HIV in 2010.Med. Trop. (Mars). 71, 6:608-12.

El-Bahnasawy, MM, DabbousHKh,Morsy, T A, 2010: Imported malaria as a threat to Egypt. J. Egypt. Soc. Parasitol. 40, 3:773-87.

El-Bahnasawy, MM, Soliman, SA, Morsy, T A, 2014:Trainingnurses on dealingwith arthropod-borne infectious diseases: Is itamandatory nowadays in Sub-Saharan-Africa? Egyptian Military Medical Journal (EMMJ) 69, 1:32-50.

El-Bahnasawy, MM, Megahed, LA, Saleh, H AA, Morsy, TA, 2015: Training program for the nursing staff regarding the viral hemorrhagic fevers (VHFs) in a fever hospital. J. Egypt. Soc. Parasitol. 45, 2:249-72

Fey, MK, Milliner, RS, 2008: A CompetencyBased Orientation Program for New Graduate Nurses. Jona Lippincott Williams \&Wilkins, Inc. Ghansah, A, Amenga-Etego, L, Amambua, N A, Andagalu, B, Apinjoh, T, et al, 2014: Monitoring parasite diversity for malaria elimination in Sub-Saharan Africa. Science 345, 6202: 1297-8

Hanafi-Bojd, AA, Vatandoost, H, Oshaghi, M, Eshraghian, M, et al, 2011: Knowledge, attitudes and practices regarding malaria control in an endemic area of southern Iran. Southeast Asian J. Trop. Med. Pub. Hlth. 42:491-501

Kanamori, S, Kohi, TW, Nyamhanga, T, Mkude, S, 2011: Assessing the performance of nurses in the management of malaria patients in Tanzania. J. Trop. Pediatr. 57, 5:378-81.

Kanamori, S, Kohi, TW, Nyamhanga, T, Mkude, S, 2011: Assessing the performance of nurses in the management of malaria patients in Tanzania. J. Trop. Pediatr. 57, 5:378-81.

Lahti, M, Hätönen, H, Välimäki, M, 2014: Impact of e-learning on nurses' and student nurses knowledge, skills, and satisfaction: A systematic review and meta-analysis. Int. J. Nurs. Stud. 51, 1:136-49.

Lynore, D, DeSilets, ED, 2007:Need assessment: An array of possibilities, J. Conti. Educa. Nursing 38, 3:107-14.

Nadjm, B, Behrens, RH, 2012: Malaria: an update for physician. Infect. Dis. Clin. North 
Am. 26, 2:243-59.

Manzi, F, Schellenberg, J, Hamis, Y, Mushi, AK, et al, 2009: Intermittent preventive treatment for malaria and anemia control in Tanzanian infants; the development and imlementation of a public health strategy. Trans. R. Soc. Trop. Med. Hyg. 103, 1:79-86.

McConnell, MM, Regehr, G, Wood, TJ, Eva, KW, 2012:Self-monitoring and its relationship to medical knowledge. Adv. Hlth. Sci. Educ. Theo. Pract. 17, 3:311-23.

Mubyazi, G, Bloch, P, Kamugisha, M, Kitua, A, Ijumba, J, 2005: Intermittent preventive treatment of malaria during pregnancy: A qualitative study of knowledge, attitudes and practices of district health managers, antenatal care staff and pregnant women in Korogwe District, North-Eastern Tanzania. Malar. J. 4:31-9.

Mushi, AK, Massaga, JJ,Mandara, CI, Mubyazi, GM,Francis, F, et al,2016: Acceptability of malaria rapid diagnostic tests administered by village health workers in Pangani District, North eastern Tanzania. Malar. J. 15, 1:439-45-z.

Nikki, K, Campos, JD, 2010: The legalities of nursing documentation. J. Men. Nurs. 40, 1:7-9.

Nsimba, SE, 2006: Assessing prescribing and patient care indicators for children under five years old with malaria and other disease conditions in public primary health care facilities. Southeast Asian J. Trop. Med. Publ. Hlth. 37, 1:206-14.

Nsimba, SE, 2007: Assessing the impact of educational intervention for improving management of malaria and other childhood illnesses in Kibaha District-Tanzania. East Afr. J. Publ. Hlth. 4, 1:5-11.

Olalekan, AW, Adebukola, AM, 2015: Effects of training on knowledge, attitude and practices of malaria prevention and control among community role model care givers in Southwestern Nigeria. Ethiop. J. Hlth. Sci. 25, 4:329-36.

Sinclair, P, Kable, A, Levett-Jones, T, 2015: The effectiveness of internet-based e-learning on clinician behavior and patient outcomes: A systematic review protocol. JBI.Database System.

Rev. Implement. Rep. 13, 1:52-64.

Singh, R, Musa J, Singh S, Ebere UV, 2014: Knowledge, attitude and practices on malaria among the rural communities in aliero, northern Nigeria. J. Family Med. Prim. Care 3, 1:39-44.

Snow, RW, Amratia, P, Kabaria, CW, Noor, AM, Marsh, K, 2012:the changing limits and incidence of malaria in Africa: 1939-2009. Adv. Parasitol. 78:169-262.

Ssekabira, U, Bukirwa, H, Hopkins, H, Namagembe, Marci, A, et al, 2008: Improved malaria case management after integrated teambased training of health care workers in Uganda. Am. J. Trop. Med. Hyg. 79, 6:826-33.

Suchitra, JB, Lakshmi Devi, N, 2007: Impact of education on knowledge, attitudes and practices among various categories of health care workers on nosocomial infections. Indian $\mathrm{J}$. Med. Micro-biol. 25, 3:181-7.

The United Republic of Tanzania, 2015: Health sector strategic plan III (July 2009-June 2015): Partnership for delivering the MDGs. Dar es Salaam: Ministry of Health \& Social Welfare. Tweed, C, Tweed, M, 2008: Intensive care nurses' knowledge of pressure ulcers: Development ofan assessment tool and effect of an educational program. Am. J. Crit. Care 17:338-46.

WHO, 2014: Health Promotion and Disease Prevention Through Population-Based Interventions, Including Action to Address Social Determinants and Health Inequity. www. emro. who. int.

WHO, 2018: WHO Recognizes National Efforts Towards Malaria Elimination In Dar es Salaam, 26-27 February 2018,Tanzania.

Wojczewski, S, Pentz, S, Blacklock, C, Hoffmann, K, Peersman, W, et al, 2015: African female physicians and nurses in the global care chain: Qualitative explorations from five destination countries. PLoS One 10, 6:e0129464.

\section{Explanations of Figures}

Fig. 1: Demographic characteristics of studied nursing staff in relation to ages.

Fig. 2: Demographic characteristics of studied nursing staff in relation to sex $(\mathrm{N}=96)$.

Fig. 3: Demographic characteristics of studied nursing staff in relation to working units .

Fig. 4: Demographic characteristics of the studied nursing staff in relation to educational qualifications.

Fig. 5: Demographic characteristics of nursing staff in relation to Years of experience in nursing.

Fig. 6: Educational needs assessment Nursing Staff regarding malaria.

Fig. 7: Nursing Staff knowledge levels regarding definition, epidemics, causes, mode of transmission, incubation period of malaria, people at risk of acquiring malaria, and affecting organs in pre and post- tests.

Fig. 8: Nursing Staff level regarding knowledge signs, symptoms, diagnosis, laboratory test, treatment and complication in pre \& post- tests. 

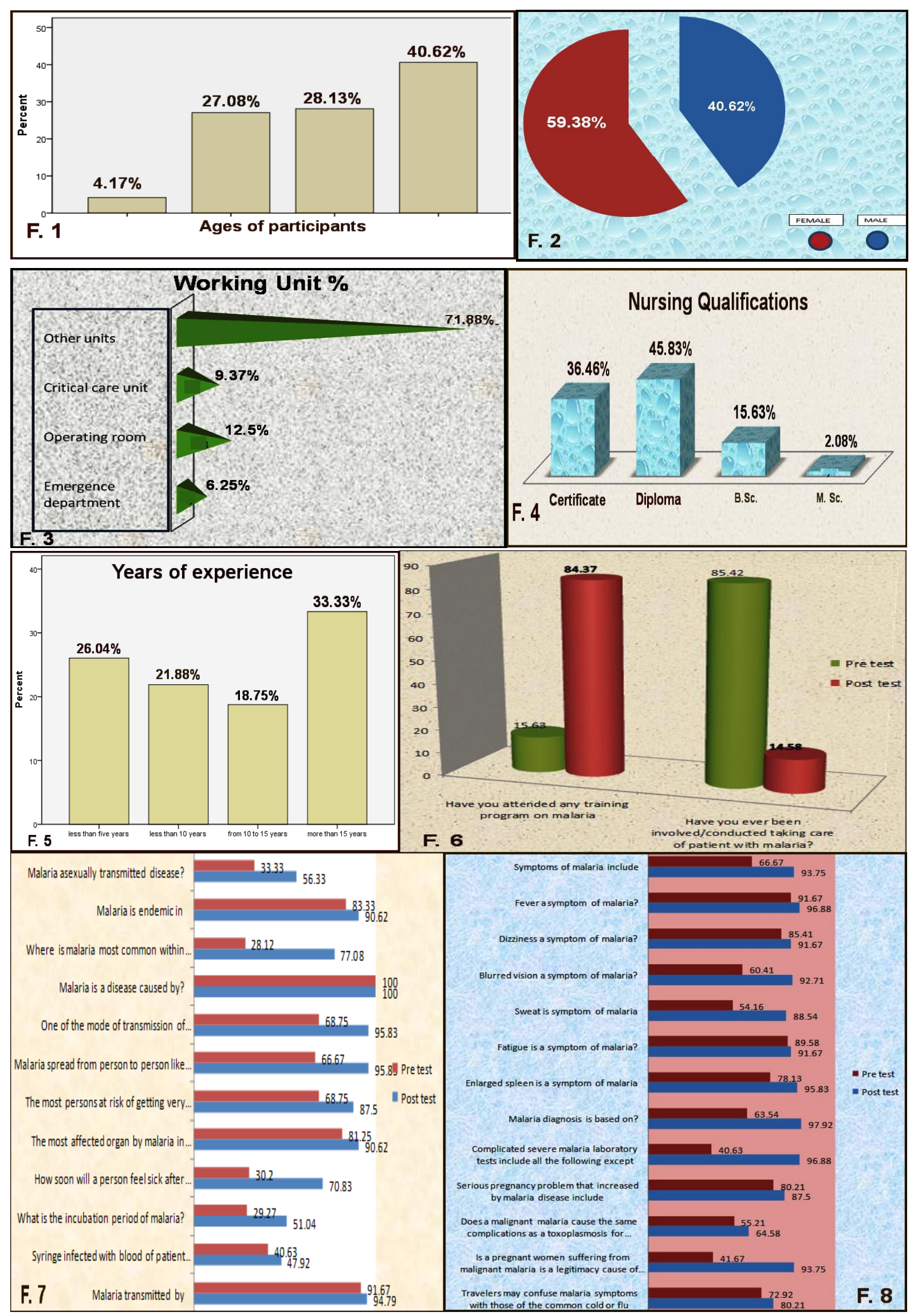